Kristo VAHER ${ }^{1}$

Tauno OTTO ${ }^{2}$

Jüri RIIVES ${ }^{2}$

\title{
POSITIONING ERROR CORRECTION OF AUTONOMOUSLY MOVABLE ROBOT ARM
}

\begin{abstract}
Industrial robots are mainly used stationarily in one working position. SMEs often find themselves in situations where robots don't have enough work to do, and because in general, robots cannot be easily moved to another position, the efficiency of robots will decrease. This study provides a solution for this issue. The solution can be found in a robot work cell where a mobile robot deals with robot arm transportation. However, since the mobile robot is not precise enough in positioning, machine vision is used to overcome this problem, which helps the robot to position itself accurately in relation to the work object. The solution has been developed and tested successfully at an Industry 4.0 testbed.
\end{abstract}

\section{INTRODUCTION}

Within all areas of robotics, the demand for collaborative and more flexible systems is rising [1]. Human-robot collaboration (HRC) has been active in the past to realize future manufacturing expectations. HRC has been made possible by several research results obtained during the past five to ten years within the scientific communities of robotics and automation [2]. Industrial robots are widely used in the industry, but there are also companies who have not been able to afford to buy robots. Mostly these are SMEs for whom buying a robot is a big investment and the investment has to pay back within a reasonable time. One of the reasons for not using a robot, according to a previous study [3], is the insufficient applicability of the robot in one working position. The company may not have enough work for the robot in one particular position in order to derive as much benefit from it as is possible. To achieve this, one would need to direct more robot operations to the robot or move the robot from one working position to another. In order to maximize the use of the robot arm in situations where there are not enough tasks in one workstation, the robot should be transported from one working position to another by

\footnotetext{
${ }^{1}$ Tallinn University of Technology (TalTech), Estonia

2 TTK University of Applied Sciences, Tallinn, Estonia

E-mail:vaher.kristo@gmail.com

https://doi.org/10.36897/jme/129013
} 
a mobile robot. The task of a mobile robot would be transporting a number of robot arms on factory level. The digitalisation of industry is redefining the role of robots - they are expected to be mobile and collaborative. However, the problem with mobile robots is their low accuracy. Paijens et al. have elaborated the approach for odometry of mobile robots through the implementation and calibration of mouse sensors [4].

I.-M. Cheng has used linear error model to determine the error parameters in his research of rapidly reconfigurable robotic work cells [5]. Fujishima et al. have researched sensing interface board to solve thermal displacement compensation [6]. Dittrich et al. have worked on autonomous machine tool using adaptive process control [7].

This article aims to solve the problem of inaccuracy in repositioning a transportable robot arm, so that at each working position the robot arm could operate within its own working accuracy. The solution is to use machine vision which can detect markers and evaluate the offset of the robot's base coordinate. The development of collaboration robots and their general compliance with Industry 4.0 principles have made the implementation of industrial robots with other products fairly easy [8-12].

\section{WORK CELL BASED ON INDUSTRY 4.0 METHODOLOGY}

In 2018, an Industry 4.0 testbed was completed at TTK University of Applied Sciences [13]. The testbed was used for practical tests within the current research. Among other system components, the testbed includes the MIR 100 mobile robot and the Universal Robots UR10 industrial robot. The concept of the laboratory provided for the construction of a fully automated production system where human presence is not necessary. The production system is divided into modules: production, post-processing, automatic warehouse, delivery of goods, intralogistics. In the latter module, the mobile robot and the collaboration robot are working together. These two machines are physically interconnected in a manner in which the mobile robot transports the robot arm between different modules (Fig. 1). Each module has specific tasks for the robot arm.

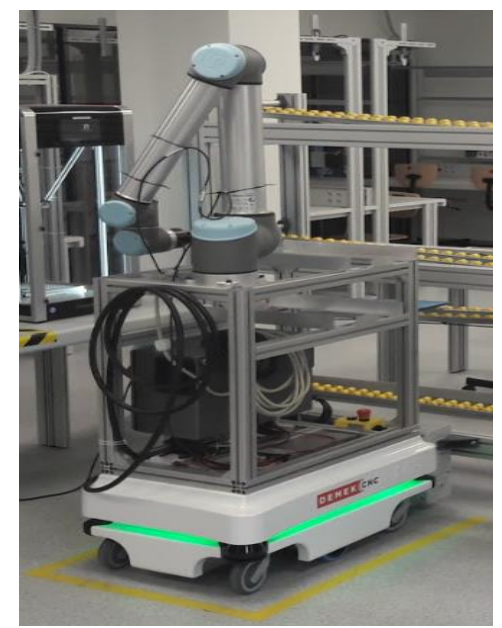

Fig. 1. The tandem of robot arm and mobile robot 
The robot tandem is able to work independently, but its work is constrained by low accuracy. The main problem is the different accuracy of the two machines. The robot arm can reposition itself within $\pm 0.05 \mathrm{~mm}$. The positioning accuracy of the mobile robot is \pm 10.0 $\mathrm{mm}$ if it is using positioning marker. Without it, the accuracy is even worse, $\pm 50.0 \mathrm{~mm}$. Due to the low accuracy class of the mobile robot, its positioning error is transmitted to the work of the robot arm and the accuracy of the robot arm equals with the accuracy of the mobile robot. This considerable difference causes a problem for the robot arm, as the robot's job is to pinpoint certain objects where the accuracy must be about $\pm 1 \mathrm{~mm}$.

The positioning accuracy of the mobile robot cannot be improved to the level of the robot arm. The solution to the problem should come from an external device or sensors. Therefore, the authors have proposed to use machine vision to detect markers and evaluate the offset from zero condition. Markers must be installed at each working position and each marker must be rigidly linked to the location where the work is to be performed. Each time the robot enters a working position, a shift in the mobile robot relative to the working position is detected.

The camera must be mounted to the robot arm close to tool location. In each working position, the robot arm moves above the marker and takes a picture of it. The difference in coordinates $(X, Y$, angle) between the position of the initially trained marker and the new position is calculated and added to the base coordinate of the robot arm. In this way, the robot arm always works with the same program and with the same precision. The positioning accuracy of the mobile robot with a marker is $\pm 10.0 \mathrm{~mm}$ - small enough in the camera's field of view to fit inside the detection area. $Z$-axis measurement is not important due to the fact that the height of the mobile robot is not changing during the positioning.

\section{RESEARCH SETUP}

The camera that was used in the experiment was Cognex IS2000M-130-40-00 with $25 \mathrm{~mm}$ lens. The camera has monochrome image recognition (black and white). The focus length is manually adjustable, which meant that the same marker was detected exactly at the same distance for all the detection points. In this project, the detection distance was set at $30 \mathrm{~cm}$.

Next to each working position, an L-shape sticker (Fig. 2) was added, rigidly connected to working stations such as the 3D printer or the parcel cabinet. L-sticker was used to define the position of the mobile robot in relation to the working station. Each time the mobile robot entered a working position, the L-sticker was captured with camera and the offset from zero position was calculated and added to the program of the robot arm.

A new program for a new location could be programmed with the UR control panel. The Camera_Start subroutine had to be chosen at the beginning of the UR program. The camera that has a manually adjustable focus point had to be positioned over a subject (300 $\mathrm{mm})$. Stop command had to be selected at this point. After that, the camera trigger subroutine had to be called. After triggering the camera, three variables (fixX, fixY and fixA) occurred, these variables had to be noted. 


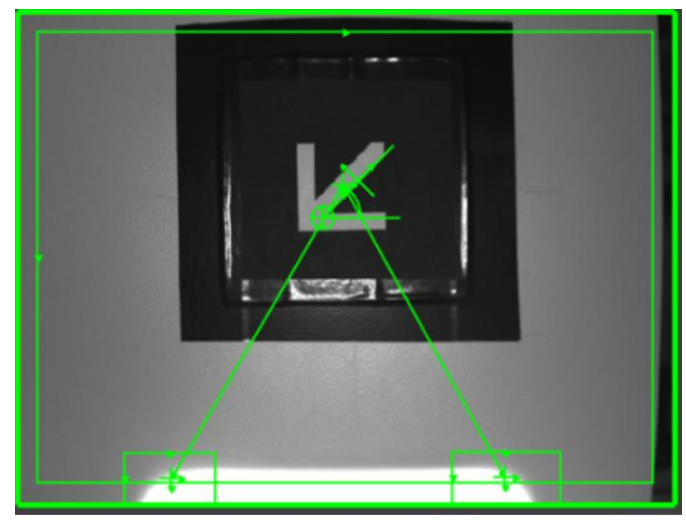

Fig. 2. L-sticker deduction with camera (In-Sight Explorer)

Because the Cognex URCAP caused the robot to stop working, the robot communicated with the RPC server, which in turn communicated with the camera via telnet. The RPC server is configured to port 555 and has 3 features:

- start_camera_telnet: This function must be executed in the UR in any program that requires communication with the camera. The function expects no values and returns either 0 (communication with the camera failed) or 1 (communication with the camera succeeded).

- change_camera_job: Function can be used to change the camera job if needed. It waits for one value (the job number) and returns one of three options - 0 (camera job exchange failed), 1 (camera job exchange succeeded), or 2 (the camera already had a job and nothing was changed).

- trigger_camera: The function can send a capture command and does not expect any value. FUNCTION returns one of three options, " $0,0,0$ " (failed to send command to camera), "1,0,0,0,0" (failed to send command to camera, the camera did not finish the job successfully), or " $1, x, y$, angle, 1 " (command was successfully transmitted to the camera, coordinates of the object were detected and the camera job succeeded). The given $X$ and $Y$ are relative to the camera.

The camera used in the current research does not have an ability to position the object in a form where we can read out $X$ - and $Y$ - coordinates. It only has the ability to detect objects and measure distances between them. Therefore, we attached an object that would always be in the same position in relation to the camera (Fig. 3).

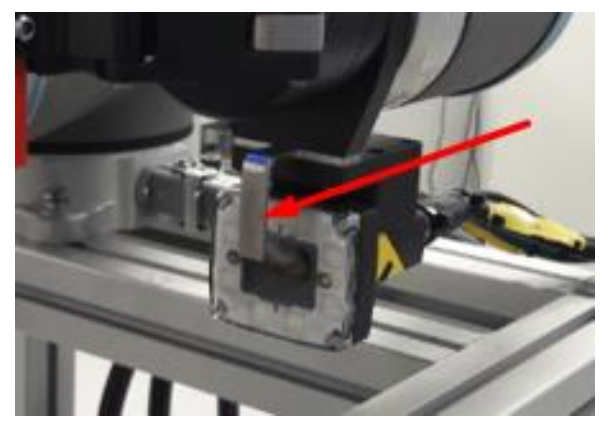

Fig. 3. Permanent object in front of camera 
The following settings were set up on the camera (Fig. 2. on the right):

- Marker recognition.

- Detecting the left corner of the subject attached to the camera.

- Detecting the right angle of the subject attached to the camera.

- Measuring the distance between the sticker and the left corner.

- Measuring distance between the sticker and the right angle.

- Measuring the angle of the camera and the subject on the camera.

If all the objects were successfully identified, the results were transmitted to the RPC server. If we got to know the distances between the angles of the object on the camera (the $X$ - and $Y$-coordinates of these angles were also known to us in advance) then we could calculate the position of the L- sticker on the camera's axis.

There are three sub-programs for using the camera:

- Camera_Start: this programme needs to be run before using other camera functions.

- Camera_Trigger: the program sends a capture command to camera and receives new $X, Y$ and angles in response.

- Camera_Change_job: changes the camera job. Variable jobID: must have the number of the corresponding job.

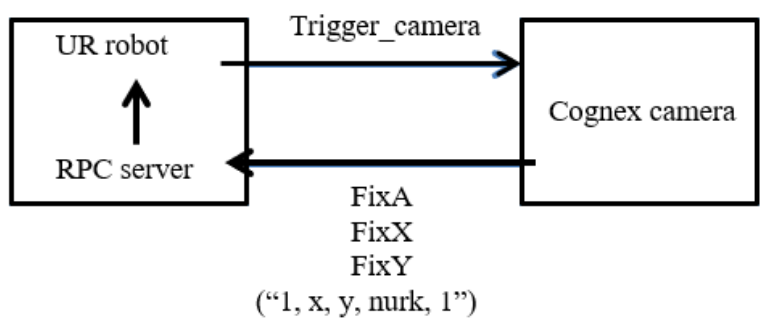

Fig. 4. Communication between camera and RPC server

To measure the positioning accuracy (ability to repeatedly position at same coordinates) of the mobile robot, a one-millimeter graph paper was placed onto the work surface, where the robot arm would make marks. The marks where made with three different positioning methods - positioning without a marker (Test \#1), positioning with a VL- marker (Test \#2), positioning with machine vision (Test \#3). The number of marks made in each position was 50. The bigger amount of marks did not give better results in terms of dispersion. The mobile robot moved between positions in an automatic mode (Fig. 5).

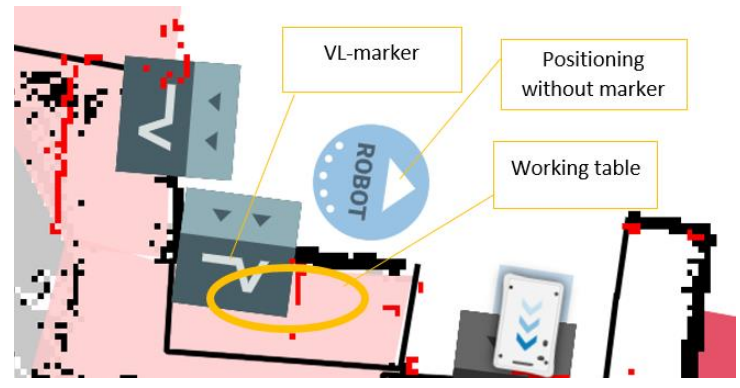

Fig. 5. A laser scanned map of the MIR robot with positioning points 
Graph paper had three different areas where markings were made. Area \# 1 (Fig. 6.) was for the robot positioning without the marker, area \# 2 was for the VL- marker positioning and area \#3 was for the machine vision positioning. All marks where measured and transferred to an excel file.

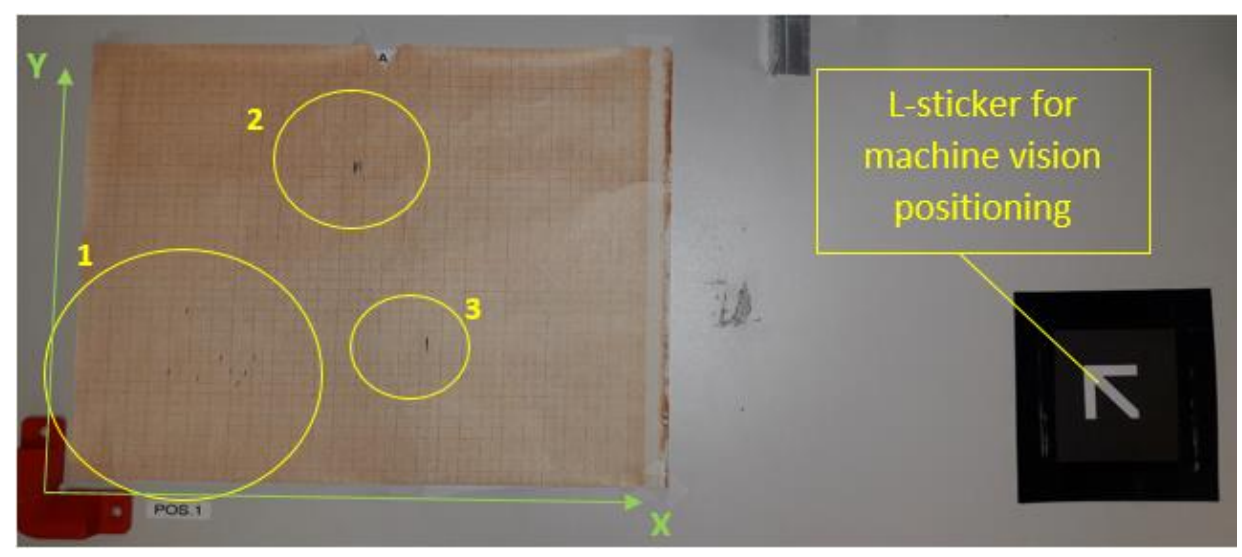

Fig. 6. Working table

For machine vision test, the following mathematical approach was given to the UR program to consider with data coming from Cognex camera:

$$
\begin{array}{rr}
\text { add } X:=\frac{\text { first } X-\text { fix } X}{1000} & \\
\text { nurk }:=(\text { first } A-f i x A) *-1 & d d y:=\frac{\text { first } Y-\text { fix } Y}{1000} \\
\text { Base_var }:=p[0,0,0,0,0, \text { nurk }] & \text { pose_add(Base_var, } p[\text { add } X, \text { add } Y, 0,0,0,0])
\end{array}
$$

Fig. 7. Angle correction formula (left), coordinates X and Y correction formula (right)

\section{POSITIONING ACCURACY ANALYSIS}

\subsection{POSITIONING WITHOUT THE MARKER}

\section{Test \# 1 - mobile robot positioning without positioning marker}

In case positioning without a positioning marking, the positioning accuracy of the mobile robot was poor. According to the information provided by the manufacturer, the positioning accuracy is $+/-50 \mathrm{~mm}$. The mobile robot positions itself with laser sensors measuring distance from surrounding objects like walls, and compares the obtained measurement with a previously stored map (all black lines on the map, Fig. 5). The result of positioning by this method was found as the result of the test in Test \# 1 (Figs. 8, 9).

The results of this experiment showed that by positioning freely on the map without a marker, the robot can reposition itself in $X$-axis $+/-50 \mathrm{~mm}$ and in $Y$-axis $+/-45 \mathrm{~mm}$. Therefore, the data given by the manufacturer is correct. 


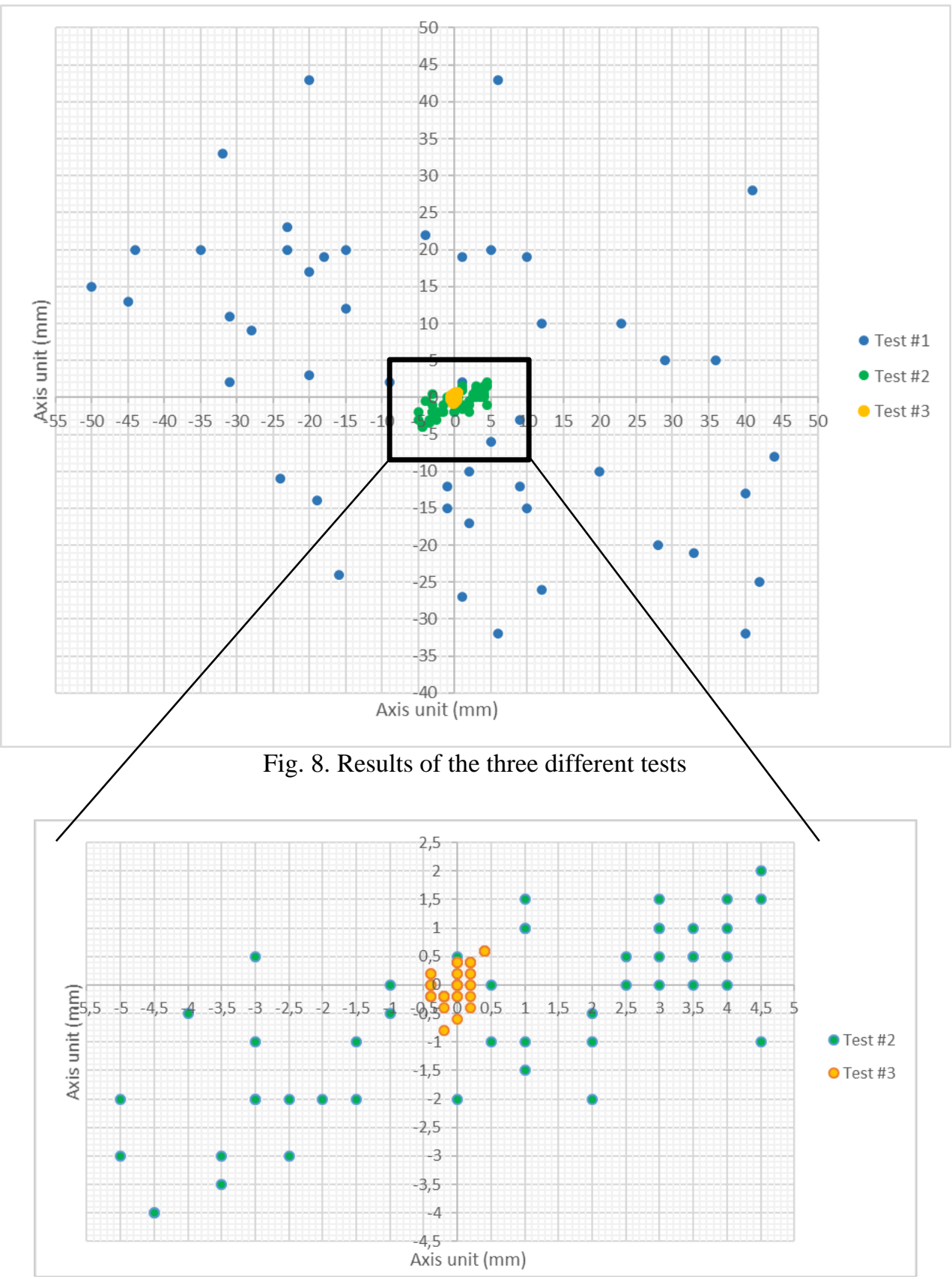

Fig. 9. Zoom in, view of two tests Fig. 8

\subsection{POSITIONING WITH VL-MARKER}

Test \# 2, mobile robot positioning with VL-marker

The VL- marker is a method provided by the manufacturer for more accurate positioning of the mobile robot. The VL- marker is basically a wall with a V-shaped cavity. The distance between the two sides of the cavity are measured constantly by the laser while approaching that cavity. The position of the mobile robot is constantly adjusted. With such a marker, according to the manufacturer's information, the robot can position itself with an accuracy of $+/-10 \mathrm{~mm}$. 
The results of the experiment showed that by using the VL- marker, the robot is able to reposition itself on the $X$-axis with an accuracy of $+/-5 \mathrm{~mm}$ and on the $Y$-axis with an accuracy of $+/-3.5 \mathrm{~mm}$.

\subsection{POSITIONING WITH MACHINE VISION}

\section{Test \# 3, robot positioning with machine vision}

Position for the machine vision is detected at the location of the mobile robot in the VL- marker. The repeatability of positioning to this marker is given in the previous chapter.

The results of the experiment showed that by using machine vision, the mobile robot is able to reposition itself on $X$-axis direction with an accuracy of $+/-0.5 \mathrm{~mm}$ and on the $Y$ axis with an accuracy of $+/-1.0 \mathrm{~mm}$.

The large error $(+/-1.0 \mathrm{~mm})$ towards $Y$-axis resulted from the setup of the machine vision parameters. This error probably came from the angle measurement of the L-sticker. It was not very precise due to the fact that pixels were converted to millimeters. This small error was also shown on the first picture, where the camera position angle was adjusted, also effecting $X$ - and $Y$ - coordinates that were taken with the next picture. Therefore, more precise measurement of the angle needs to be established to correct the conversion of measures.

\section{CONCLUSION}

In earlier work on robotised work cells [3], the reasons were found that prevent SMEs from adopting robots, and various variants of robot implementation in enterprises were proposed [3]. It was found that the best solution for SMEs is to obtain a robot that can be moved from one job position to another at the factory level, and this should be done with an automated mobile robot.

Adding a Cognex camera to the Universal Robots robot arm is technically easy and requires little programming. Cognex and UR work well together even though the Cognex application for the UR robot interface is inapplicable. This issue was resolved by using scripts written to the server of the UR robot controller. Scripts were triggered in the UR program when needed.

Machine vision significantly improved the accuracy of the robot arm despite the inadequate positioning accuracy of the mobile robot. With machine vision, a repeat accuracy of $+/-0.5 \mathrm{~mm}$ for the robot arm was achieved. The robot arm itself has an accuracy of $+/-0.05 \mathrm{~mm}$, but the result of using machine vision was sufficient enough to perform most of the tasks.

The further aim of work on robotised work cells is to achieve a result where the robot arm would be separated from the mobile robot and both machines would be capable of operating independently from each other. The task of the mobile robot would be to transport several robotic arms on factory level. In the scope ot this article, the problem of inaccuracy in repositioning a transportable robot arm was solved: in each working 
position, the robotic arm could work within its own accuracy regardless of how accurately it was trasnported to the working position.

\section{REFERENCES}

[1] EUROBOTICS., 2013, Robotics 2020, Strategic Research Agenda for Robotics in Europa. https://www.eurobotics.net/cms/upload/topic_groups/SRA2020_SPARC.pdf

[2] WANG L., GAO R., VANCZA J., 2019, Symbiotic Human-Robot Collaborative Assembly, CIRP Ann., 68/2, 701-726.

[3] VAHER K., KANGRU T.; OTTO T., RIIVES J., 2019, The Mobility of Robotised Work Cells in Manufacturing, Proceedings of the 30th International DAAAM Symposium, Intelligent Manufacturing \& Automation: 23-26th October 2019, Zadar, Croatia.

[4] PAIJENS A.F.M., HUANG L., AL-JUMAILY A.M., 2020, Implementation and Calibration of an Odometry System for Mobile Robots, Based on Optical Computer Mouse Sensors, Sensors and Actuators A: Physical, 301, 111731.

[5] CHEN I.M., 2001, Rapid Response Manufacturing Through a Rapidly Reconfigurable Robotic Workcell. Robotics and Computer-Integrated Manufacturing, 17/3, 199-213.

[6] FUJISHIMA M., MORI M., NARIMATSU K., IRINO N., 2019, Utilisation of IoT and Sensing for Machine Tools, Journal of Machine Engineering, 19/1, 38-47.

[7] DITTRICH M.A., DENKENA B., HAYTHEM B., UHLICH, F., 2019, Autonomous Machining - Recent Advances in Process Planning and Control, Journal of Machine Engineering, 19/1, 28-37.

[8] LU Y., 2017, Industry 4.0: A Survey on Technologies, Journal of Industrial Information Integration, Applications and Open Research, 6, 1-10, 10.1016/j.jii.2017.04.005.

[9] ROBLA-GOMEZ S., BECERRA V.M., LLATA J.R., 2017, Working Together: A Review on Safe Human-Robot Collaboration in Industrial Environments, Journals \& Magazines IEEE Access. 5., 10.1109/ACCESS.2017. 2773127.

[10] LOUN K., RIIVES J., OTTO T., 2012, Workplace Performance and Capability Optimization in the Integrated Manufacturing, Proceedings of 8th International Conference of DAAAM Baltic Industrial Engineering, Tallinn.

[11] KUTS V., OTTO T., TÄHEMAA T., BONDARENKO Y., 2019, Digital Twin Based Synchronised Control and Simulation of the Industrial Robotic Cell Using Virtual Reality, Journal of Machine Engineering, 19/1, 128-145.

[12] SELL R., OTTO T., 2008, Remotely Controlled Multi Robot Environment, Proceedings of 19th EAEEIE Annual Conference, 20-25.10.1109/EAEEIE.2008.4610152., Tallinn.

[13] VAHER K., VAINOLA V., OTTO T., 2019, Industry 4.0 Laboratory, Industry 4.0, Technological Basis of Industy 4.0, 1/5, Proceedings IV International Scientific Conference, 24-27.06, Burgas, Bulgaria. 